\title{
An Efficient ELM Approach for Blood Vessel Segmentation in Retinal Images
}

\author{
X. Merlin Sheeba and S. Vasanthi
}

\begin{abstract}
Diabetic Retinopathy (DR) is one of the most important ophthalmic pathological reasons of blindness among people of working age. Previous techniques for blood vessel detection in retinal images can be categorized into rulebased and supervised methods. This research presents a new supervised technique for blood vessel detection in digital retinal images. This novel approach uses an Extreme Learning Machine (ELM) approach for pixel classification and calculates a 7-D vector comprises of gray-level and moment invariants-based features for pixel representation. The approach is based on pixel classification using a 7-D feature vector obtained from preprocessed retinal images and given as input to a ELM. Classification results (real values between 0 and 1) are thresholded to categorize each pixel into two classes namely vessel and nonvessel. Ultimately, a post processing fills pixel gaps in detected blood vessels and eliminates falsely-detected isolated vessel pixels. The technique was assessed on the publicly available DRIVE and STARE databases, widely used for this purpose, as they comprises of retinal images where the vascular structure has been precisely marked by experts. Method performance on both sets of test images is better than other existing solutions in literature. The approach proves particularly accurate for vessel detection in STARE images. Its efficiency and strength with different image conditions, along with its simplicity and fast implementation, make this blood vessel segmentation proposal appropriate for retinal image computer analyses such as automated screening for early diabetic retinopathy detection.
\end{abstract}

Keywords - Diabetic Retinopathy, Moment Invariants, Retinal Imaging, Vessels Segmentation

\section{INTRODUCTION}

D IABETIC retinopathy (DR) is one of the most serious and most frequent eye diseases in the world; it is the most common cause of blindness [2] [3] in adults between 20 and 60 years of age. DR is a silent disease in its first stages, i.e. many patients are not aware of its presence, it often remains undiagnosed until serious vision impairment occurs. To build an expert system that is able to perform the diagnoses task needed to use digital retinal images. By imaging the retina of a person with a special camera, then using image processing and pattern recognition technique to analyze that retina make a specific diagnoses decision. One of the important operations

X. Merlin Sheeba, KS Rangasamy College of Tehnology, Tiruchengode, Email: renachristina@gmail.com

S. Vasanthi, KS Rangasamy College of Tehnology, Tiruchengode performed on the digital retina is the edge detection of blood vessels of the retina. A specialized edge detection technique may be needed since traditional edge detection technique might not be able to accurately segment the vessels from the background of the retinal image. Accurate vasculature segmentation is a difficult task for several reasons: the presence of noise, the low contrast between vessels and background and the variability of vessels width, brightness, and shape.

Several methods have been proposed for this purpose, matched filer, morphology edge detection, and ridge -based vessel segmentation which is called pixel classification, tracking methods, supervised classification and wavelet transform. Also, due to the reflection on the tiny uneven surface of the soft tissue in the image, the low contrast between the vessels and background and the pathological variations, detecting blood vessels automatically from a retinal image is a challenging problem. Many methods have been used for retinal vessel segmentation. These can be divided into two groups: rule-based methods and supervised method. Supervised methods are based on pixel classification, which consists on classifying each pixel into two classes, vessel and non-vessel. Regarding rule-based methods, vessel tracking method attempt to obtain the vasculature structure by following vessel center lines.

The segmentation of vessels can be represented in two ways: One way is to mark out all vessel pixels. The other way is to find the vessel centerlines and the radius at each pixel of the center lines. The main aim of vessel segmentation algorithm is automated detection of eye diseases.

\section{LITERATURE SURVEY}

Currently there are number of automatic systems has been developed for the detection of various eye diseases like diabetic retinopathy. To segment vessels in retinal images, seven classes of methods have been commonly used matched filters, vessel tracking, morphological processing, region growing, multiscale, supervised and adaptive thresholding approaches. Some of the reference papers have been explained below;

Ricci and perfetti [4] uses basic line detector whose response is thresholded to obtain unsupervised pixel classification. They employ two orthogonal line detectors along with the grey level of the target pixel to construct feature vector for supervised classification using a support vector machine. The basic line detector; is computationally simple and gives very good result with respect to existing unsupervised methods. SVM guarantee good generalization performance even with a small training set. Performance 
estimated both in terms of AUC and accuracy show this supervised system is slightly superior to other existing system. Some false positive are found around the border of the optic disk and in proximity of the pathological regions. Some problems occur to segment the thinnest vessels or when the local contrast is quite low, in this case, vessels can be captured only at the expense of high FPR. Because of this, segmentation image tends to be quite noisy. Maria mendonca [5] combines the retinal vascular network with differential filters, for vessel centerline extraction with morphological operators, used for filling vessel segments. Several intensity and morphological properties of vascular structures, such as linearity, connectivity, and width are considered. The main type of errors are 1)detection of other retinal structure, like the optic disc, several pathological areas and background structures,2) under segmentation of some vessel segments,3) partial or complete missing if thin vessel branches. Staal [6] used a system which is based on extraction of image ridges, which coincide approximately with vessel centerlines. The ridges are used to compose primitives in the form of line elements. With the line elements an image is partitioned into patches by assigning each image pixel to the closest line element. Every line element constitutes a local coordinate frame for its corresponding patch. For every pixel, feature vectors are computed that make use of properties of the patches and the line elements. The feature vectors are classified using a NNclassifier and sequential forward feature selection. Two types of errors can be distinguished. The first type is over- and under segmentation of the vessels. This is important in applications where determination of the vessel width is needed. The second type of error is the missing or erroneous detection of vessel branches. Marin [1] proposed a new methodology for blood vessel detection is presented. It is based on pixel classification using a 7-D feature vector extracted from preprocessed retinal images and given as input to a neural network. The NN was then fed with the values of these pixel windows for classifying each pixel into vessel or not. Classification results are thresholded to classify each pixel into two classes: vessel and nonvessel. Classifiers are trained by supervised learning with data from manually-labeled images. Finally, a post processing fills pixel gaps in detected blood vessels and removes falselydetected isolated vessel pixels. Despite of its simplicity, the high accuracy achieved by this method in blood vessel detection.

\section{MATERIAL}

To evaluate the vessel segmentation methodology described in the next section, two publicly available databases containing retinal images, the DRIVE [8] and STARE [9] databases, were used. These databases have been widely used by other researchers to test their vessel segmentation methodologies since,apart from being public, they provide manual segmentations for performance evaluation. The DRIVE database comprises 40 eye-fundus color images (seven of which present pathology) taken with a Canon CR5 nonmydriatic 3CCD camera with a 45 field-of-view (FOV). Each image was captured at 768 X 584 pixels; 8 bits per color plane and, in spite of being offered in LZW compressed TIFF format, they were originally saved in JPEG format. The database is divided into two sets: a test set and a training set, each of them containing 20 images. The test set provides the corresponding FOV masks for the images, which are circular (approximated diameter of 540 pixels) and two manual segmentations generated by two different specialists for each image. The selection of the first observer is accepted as ground truth and used for algorithm performance evaluation in literature. The training set also includes the FOV masks for the images and a set of manual segmentations made by the first observer. The STARE database, originally collected by Hoover et al. [7], comprises 20 eye-fundus color images (ten of them contain pathology) captured with a TopCon TRV-50 fundus camera at $35 \mathrm{FOV}$. The images were digitalized to 700 $\mathrm{X} 605$ pixels, 8 bits per color channel and are available in PPM format. The database contains two sets of manual segmentations made by two different observers. Performance is computed with the segmentations of the first observer as ground truth.

\section{Vessel Segmentation Method}

This paper proposes a new supervised approach for blood vessel detection based on a ELM for pixel classification. The necessary feature vector is computed from preprocessed retinal images in the neighborhood of the pixel under consideration. The following process stages may be identified: 1) original fundus image preprocessing for gray-level homogenization and blood vessel enhancement, 2) feature extraction for pixel numerical representation, 3) application of a classifier to label the pixel as vessel or nonvessel, and 4) post processing for filling pixel gaps in detected blood vessels and removing falsely-detected isolated vessel pixels. Input images are monochrome and obtained by extracting the green band from original RGB retinal images. The green channel provides the best vessel-background contrast of the RGB-representation, while the red channel is the brightest color channel and has low contrast, and the blue one offers poor dynamic range. Thus, blood containing elements in the retinal layer (such as vessels) are best represented and reach higher contrast in the green channel [10]. All parameters described below were set by experiments carried out on DRIVE images with the aim of contributing the best segmentation performance on this database Therefore, they refer to retinas of approximately 540 pixels in diameter. The application of the methodology to retinas of different size (i.e., the diameter in pixels of STARE database retinas is approximately 650 pixels) demands either resizing input images to fulfil this condition or adapting proportionately the whole set of used parameters to this new retina size.

\section{A. Preprocessing}

Color fundus images often show important lighting variations, poor contrast and noise. In order to reduce these imperfections and generate images more suitable for extracting the pixel features demanded in the classification step, a preprocessing comprising the following steps is applied: 1) vessel central light reflex removal, 2) background homogenization, and 3) vessel enhancement. Next, a description of the procedure, illustrated through its application 
to a STARE database fundus image (Fig. 1), is detailed.

1) Vessel Central Light Reflex Removal: Since retinal blood vessels have lower reflectance when compared to other retinal surfaces, they appear darker than the background. Although the typical vessel cross-sectional gray-level profile can be approximated by a Gaussian shaped curve (inner vessel pixels are darker than the outermost ones), some blood vessels include a light streak (known as a light reflex) which runs down the central length of the blood vessel. To remove this brighter strip, the green plane of the image is filtered by applying a morphological opening using a three-pixel diameter disc, defined in a square grid by using eight-connexity, as structuring element. Disc diameter was fixed to the possible minimum value to reduce the risk of merging close vessels. denotes the resultant image for future references. An example of vessel central light reflex and its removal from a fundus image by means of opening filtering operation is shown in Fig. 1(a) .

2) Background Homogenization: Fundus images often contain background intensity variation due to nonuniform illumination. Consequently, background pixels may have different intensity for the same image and, although their graylevels are usually higher than those of vessel pixels (in relation to green channel images), the intensity values of some background pixels is comparable to that of brighter vessel pixels. Since the feature vector used to represent a pixel in the classification stage is formed by gray-scale values, this effect may worsen the performance of the vessel segmentation methodology. With the purpose of removing these background lightening variations, a shade-corrected image is accomplished from a background estimate. This image is the result of a filtering operation with a large arithmetic mean kernel, as described below.

Firstly, a 3x3 mean filter is applied to smooth occasional salt-and-pepper noise. Further noise smoothing is performed by convolving the resultant image with a Gaussian kernel of dimensions $\mathrm{mxm}=9 \times 9$, mean $\mu=0$ and variance $\sigma^{2}=1.8^{2}$, Secondly, a background image IB, is produced by applying a 69x69 mean filter [Fig. 1(b)]. When this filter is applied to the pixels in the FOV near the border, the results are strongly biased by the external dark region. To overcome this problem, out-of-the FOV gray-levels are replaced by average graylevels in the remaining pixels in the square. Then, the difference D between $I_{\gamma}$ and $I_{B}$ is calculated for every pixel

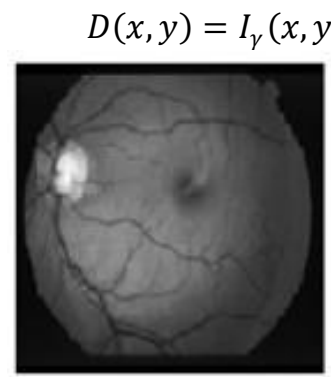

(a)

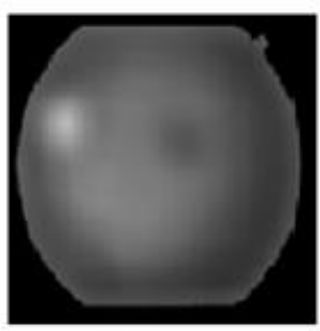

(b)

Figure 1: Illustration of the Preprocessing Process: (a) Green Channel of the Original Image. (b) Background Image
Finally, a shade-corrected image $I_{S c}$ is obtained by transforming linearly values into integers covering the whole range of possible gray-levels $([0-255]$, referred to 8-bit images). Fig. 1(d) shows the corresponding to a nonuniformly illuminated image. The proposed shade-correction algorithm is observed to reduce background intensity variations and enhance contrast in relation to the original green channel image.Besides the background intensity variations in images, intensities can reveal significant variations between images due to different illumination conditions in the acquisition process.

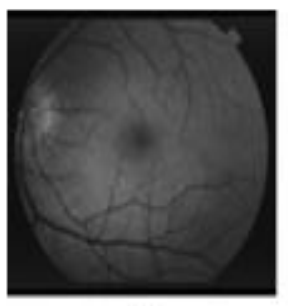

(a)

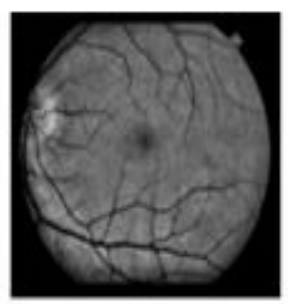

(b)

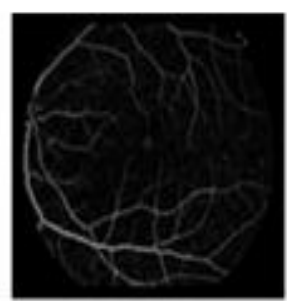

(c)
Figure 2: Two Examples of Application of the Preprocessing on Two Images with Different Illumination Conditions. (a),

Green channel of the Original Images. (b), Homogenized Images. (c), Vessel-Enhanced Images

In order to reduce this influence, a homogenized image [Fig. 1(a)] is produced as follows: the histogram of is displaced toward the middle of the gray-scale by modifying pixel intensities according to the following gray-level global transformation function:

$$
g_{\text {oUTPUT }}=\left\{\begin{array}{c}
0, \text { if } g<0 \\
255, \text { if } g>255 \\
g, \text { otherwise }
\end{array}\right\}
$$

Where

$$
g=g_{I N P U T}+128-g_{\text {INPUT_MAX }}
$$

and $g_{\text {INPUT }}$ and $g_{\text {OUTPUT }}$ are the gray-level variables of input and output images (and, respectively). The variable denoted by defines the gray-level presenting the highest number of pixels in $(x, y)$. By means of this operation, pixels with graylevel, which are observed to correspond to the background of the retina, are set to 128 for 8 -bit images. Thus, background pixels in images with different illumination conditions will standardize their intensity around this value. Fig. 2 (a), (b) and (d), (e), shows this effect for two fundus images in the STARE database.

3) Vessel Enhancement: The final preprocessing step consists on generating a new vessel-enhanced image, which proves more suitable for further extraction of moment invariants- based features (see Section IV-B).

Vessel enhancement is performed by estimating the complementary image of the homogenized image, $I_{H}^{C}$, and 
subsequently applying the morphological Top-Hat transformation [Fig. 1(f)]

$$
I_{V E}=I_{H}^{C}-\gamma\left(I_{H}^{C}\right)
$$

where is a morphological opening operation using a disc of eight pixels in radius. Thus, while bright retinal structures are removed (i.e., optic disc, possible presence of exudates or reflection artifacts), the darker structures remaining after the opening operation become enhanced (i.e., blood vessels, fovea, possible presence of microaneurysms or hemorrhages).

Samples of vessel enhancement operation results are shown in Fig. 2(c) and (f) for two fundus images with variable illumination conditions.

\section{B. Feature Extraction}

The aim of the feature extraction stage is pixel characterization by means of a feature vector, a pixel representation in terms of some quantifiable measurements which may be easily used in the classification stage to decide whether pixels belong to a real blood vessel or not. In this paper, the following sets of features were selected.

- Gray-level-based features: features based on the differences between the gray-level in the candidate pixel and a statistical value representative of its surroundings.

- Moment invariants-based features: features based on moment invariants for describing small image regions formed by the gray-scale values of a window centered on the represented pixels.

Gray-Level-Based Features: Since blood vessels are always darker than their surroundings, features based on describing gray-level variation in the surroundings of candidate pixels seem a good choice. A set of gray-level-based descriptors taking this information into account were derived from homogenized images considering only a small pixel region centered on the described pixel $(x, y) . S_{x, y}^{w}$ stands for the set of coordinates in a $w \times w$ sized square window centered on point $(x, y)$. Then, these descriptors can be expressed as

$$
\begin{gathered}
f_{1}(x, y)=I_{H}(x, y)-\min _{(s, t) \in S_{x, y}^{g}}\left\{I_{H}(s, t)\right\} \\
f_{2}(x, y)=\min _{(s, t) \in S_{x, y}^{g}}\left\{I_{H}(s, t)-I_{H}(x, y)\right\} \\
f_{3}(x, y)=I_{H}(x, y)-\min _{(s, t) \in S_{x, y}^{g}}\left\{I_{H}(s, t)\right\} \\
f_{4}(x, y)=\operatorname{std}_{(s, t) \in S_{x, y}^{g}}\left\{I_{H}(s, t)\right\} \\
f_{5}(x, y)=I_{H}(x, y)
\end{gathered}
$$

2) Moment Invariants-Based Features: The vasculature in retinal images is known to be piecewise linear and can be approximated by many connected line segments. For detecting these quasi-linear shapes, which are not all equally wide and may be oriented at any angle, shape descriptors invariant to translation, rotation and scale change may play an important role. Within this context, moment invariants proposed by $\mathrm{Hu}$
[60] provide an attractive solution and are included in the feature vector. In this paper, they are computed as follows.

Given a pixel $(\mathrm{x}, \mathrm{y})$ of the vessel-enhanced image $I_{V E}$, a subimage is generated by taking the region defined by $S_{x, y}^{17}$. The size of this region was fixed to $17 \times 17$ so that, considering that the region is centered on the middle of a "wide" vessel (89-pixel wide and referred to retinas of approximately 540 pixels in diameter), the subimage includes an approximately equal number of vessel and nonvessel pixels.

A set of seven moment invariants under size, translation, and rotation, known as $\mathrm{Hu}$ moment invariants, can be derived from combinations of regular moments. Among them, our tests have revealed that only those defined by

$$
\begin{gathered}
\phi_{1}=\eta_{20}+\eta_{02} \\
\phi_{2}=\left(\eta_{20}+\eta_{02}\right)^{2}+4 \eta_{11}^{2}
\end{gathered}
$$

constitute the combination providing optimal performance interms of average accuracy (see Section V-B). The inclusion of the remainder moments result in decreasing classification performance and increasing computation needed for classification.

Moreover, the module of the logarithm was used instead of its values themselves. Using the logarithm reduces the dynamic range and the module prevents from having to deal with the complex numbers resulting from computing the logarithm of negative moment invariants.

In conclusion, the following descriptors were considered to be part of the feature vector of a pixel located at

$$
\begin{aligned}
& f_{6}(x, y)=\left|\log \left(\phi_{1}\right)\right| \\
& f_{7}(x, y)=\left|\log \left(\phi_{2}\right)\right|
\end{aligned}
$$

where $\phi_{1}$ and $\phi_{2}$ are the moment invariants

\section{Classification}

In the feature extraction stage, each pixel from a fundus image is characterized by a vector in a 7-D feature space.

$$
F(x, y)=\left(f_{1(x, y)}, \ldots, f_{7}(x, y)\right)
$$

Now, a classification procedure assigns one of the classes (vessel) or (nonvessel) to each candidate pixel when its representation is known. In order to select a suitable classifier, the distribution of the training set data (described below) in the feature space was analyzed. The results of this analysis showed that the class linear separability grade was not high enough for the accuracy level required for vasculature segmentation in retinal images. Therefore, the use of a non linear classifier was necessary. The following nonlinear classifiers can be found in the existing literature on this topic: the kNN method [6] and [11], support vector machines [17], Bayesian classifier [12], or neural networks [13], [14]. Extreme Learning Machine classifier was selected in this paper.

\section{Extreme Learning Machine}

ELMs parameters can be analytically determined rather 
than being tuned. This algorithm provides good generalization performance at very fast learning speed. From function approximation point of view ELM[15] is very different compared to the traditional methods. ELM shows that the hidden node parameters can be completely independent from the training data.

- In conventional learning theory, the hidden node parameters cannot be created without seeing the training data.

- In ELM, the hidden node parameters can be generated before seeing the training data.

\section{Salient Features of ELM}

Compared to popular Back propagation (BP) Algorithm and Support Vector Machine (SVM), ELM has several salient features:

- Ease of use: Except predefined network architecture, no other parameters need to be manually tuned. Users need not have spent much time in tuning and training learning machines.

- Faster learning speed: The time taken for most of the training will be in milliseconds, seconds, and minutes. Other conventional methods cannot provide such a fast learning speed.

- Higher generalization performance: The generalization performance of ELM is better than SVM and back propagation in most cases.

- Applicable for all nonlinear activation functions: Discontinuous, differential, non-differential functions can be used as activation functions in ELM.

- Applicable for fully complex activation functions: Complex functions can also be used as activation functions in ELM.

Extreme Learning Machine (ELM) meant for Single Hidden Layer Feed-Forward Neural Networks (SLFNs) will randomly select the input weights and analytically determines the output weights of SLFNs. This algorithm tends to afford the best generalization performance at extremely fast learning speed.

The structure of ELM network is shown in figure 1. ELM contains an input layer, hidden layer and an output layer. The ELM has several interesting and significant features different from traditional popular learning algorithms for feed forward neural networks. These include the following:

- The learning speed of ELM is very quick when compared to other classifier. The learning process of ELM can be performed in seconds or less than seconds for several applications. In all the previous existing learning techniques, the learning performed by feed-forward network will take huge time even for simple applications.

- The ELM has enhanced generalization result when compared to the gradient-based learning techniques. The existing gradient-based learning technique and a few other learning techniques may encounter several problems like local minima, not proper learning rate and over fitting, etc. To overcome these problems, some techniques like weight decay and early stopping techniques must be utilized in these existing learning techniques.

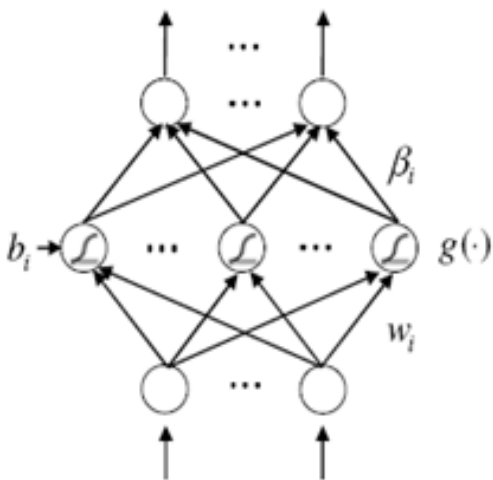

Figure 3: Structure of ELM Network

- The ELM will attain the results directly without such difficulties. The ELM learning algorithm is much simple than the other learning techniques for feed-forward neural networks. The existing learning techniques can be applied to only differentiable activation functions, whereas the ELM learning algorithm can also be used to train SLFNs with many non-differentiable activation functions.

\section{E. Extreme Learning Machine Training Algorithm}

If there are $\mathrm{N}$ samples $\left(\mathrm{x}_{\mathrm{i}}, \mathrm{t}_{\mathrm{i}}\right)$, where $\mathrm{x}_{\mathrm{i}}=\left[\mathrm{x}_{\mathrm{i} 1}, \mathrm{x}_{\mathrm{i} 2} \ldots \mathrm{x}_{\mathrm{in}}\right]^{\mathrm{T}}$ $\in \mathrm{R}^{\mathrm{n}}$ and $\mathrm{t}_{\mathrm{i}}=\left[\mathrm{t}_{\mathrm{i} 1}, \mathrm{t}_{\mathrm{i} 2}, \ldots, \mathrm{t}_{\mathrm{im}}\right]^{\mathrm{T}} \in \mathrm{R}^{\mathrm{n}}$, then the standard SLFN[16] with $\mathrm{N}$ hidden neurons and activation function $\mathrm{g}(\mathrm{x})$ is defined as:

$$
\sum_{i=1}^{\bar{N}} \beta_{i} g\left(w_{i} . x_{j}+b_{i}\right)=0_{j}, j=1, \ldots \ldots, N_{.,}
$$

where $\mathrm{w}_{\mathrm{i}}=\left[\mathrm{w}_{\mathrm{i} 1}, \mathrm{w}_{\mathrm{i} 2}, \ldots, \mathrm{w}_{\mathrm{in}}\right]^{\mathrm{T}}$ represents the weight vector that links the ith hidden neuron and the input neurons, $\beta_{i}=\left[\beta_{i 1}\right.$, $\left.\beta^{\mathrm{i} 2}, \ldots, \beta^{\mathrm{im}}\right]^{\mathrm{T}}$ represents weight vector that links the ith neuron and the output neurons, and $b_{i}$ represents the threshold of the ith hidden neuron. The "." in $\mathrm{w}_{\mathrm{i}} \cdot \mathrm{x}_{\mathrm{j}}$ indicates the inner product of $w_{i}$ and $x_{j}$. The SLFN try to reduce the difference between $o_{j}$ and $t_{\mathrm{j}}$. This can be given as:

$$
\sum_{i=1}^{\bar{N}} \beta_{i} g\left(w_{i} . x_{j}+b_{i}\right)=t_{j}, j=1, \ldots \ldots, N .,
$$

or, more in a matrix format as $\mathrm{H} B=\mathrm{T}$, where

$$
\begin{gathered}
H\left(a_{1}, \ldots, a_{\widetilde{N}}, b_{i}, \ldots, b_{\widetilde{N}}, x_{1}, \ldots, x_{N}\right) \\
=\left[\begin{array}{ccc}
g\left(w_{1}, x_{1}+b_{1}\right) & \cdots & g\left(w_{g}, x_{g}+b_{g}\right) \\
\vdots & \ddots & \vdots \\
g\left(w_{1}, x_{1}+b_{1}\right) & \cdots & g\left(w_{g}, x_{g}+b_{g}\right)
\end{array}\right]_{N * \widetilde{N}} \\
\beta=\left[\begin{array}{c}
\beta_{1}^{T} \\
\vdots \\
\beta_{\widetilde{N}}^{T}
\end{array}\right]_{\widetilde{N} * m} \\
\end{gathered}
$$

The matrix $\mathrm{H}$ is the hidden layer output matrix of the neural network. If the number of neurons in the hidden layer is equal to the number of samples, then $\mathrm{H}$ is square and invertible. Otherwise, the system of equations requires to be solved by numerical methods, concretely by solving 


$$
\min _{\beta}\|H \beta-T\|
$$

The result that reduces the norm of this least squares equation is

$$
\hat{\beta}=H^{+} T
$$

where $\mathrm{H}^{\dagger}$ is known as Moore-Penrose generalized inverse. The most significant properties of this result are:

- Minimum training error.

- Smallest norm of weights and best generalization performance.

- The minimum norm least-square solution of $\mathrm{H} \beta=\mathrm{T}$ is unique.

The ELM algorithm works as follows

Given a training set $N=\left\{\left(x_{1}, t_{1}\right) \mid x_{1} \in R^{n}, t_{1} \in R^{m}\right.$,

$1=1 \ldots \ldots . N\}$ activation function $\mathrm{g}(\mathrm{x})$ and hidden neuron $\widetilde{N}$, do the following

- Assigning random value to the input weight $w_{i}$ and the bias $b_{i}, i=1, \ldots \ldots \ldots . \widetilde{N}$

- Find the hidden layer output matrix $\mathrm{H}$.

- Find the output weight $\beta$, using $\beta^{\wedge}=\mathrm{H}^{+} \mathrm{T}$, where $\beta, \mathrm{H}$ and T are defined in the same way they were defined in the SLFN specification above.

\section{EXPERIMENTAL RESUlTS}

\section{A. Performance Measures}

In order to quantify the algorithmic performance of the proposed method on a fundus image, the resulting segmentation is compared to its corresponding gold-standard image. This image is obtained by manual creation of a vessel mask in which all vessel pixels are set to one and all nonvessel pixels are set to zero. Thus, automated vessel segmentation performance can be assessed. Table 1 shows the contingency vessel classification.

Table 1: Contingency Vessel Classification

\begin{tabular}{|c|c|c|}
\hline & Vessel Present & Vessel Absent \\
\hline Vessel Detected & $\begin{array}{c}\text { True Positive } \\
\text { (TP) }\end{array}$ & $\begin{array}{c}\text { False Positive } \\
\text { (FP) }\end{array}$ \\
\hline Vessel Not Detected & $\begin{array}{c}\text { False Negative } \\
\text { (FN) }\end{array}$ & $\begin{array}{c}\text { True Negative } \\
\text { (TN) }\end{array}$ \\
\hline
\end{tabular}

Table 2 shows the performance results on the drive database. Ten images are taken as samples for the experimental result. It is observed that the proposed approach provides significant performance.

Table 2: Performance Results On Drive Database Images

\begin{tabular}{|c|c|c|c|c|c|}
\hline Image & Se & Sp & Ppv & Npv & Acc \\
\hline 1. & 0.8252 & 0.9651 & 0.8254 & 0.9668 & 0.9721 \\
\hline 2. & 0.8145 & 0.9521 & 0.8233 & 0.9596 & 0.9845 \\
\hline 3. & 0.8123 & 0.9625 & 0.8217 & 0.9651 & 0.9736 \\
\hline 4. & 0.8145 & 0.9823 & 0.8265 & 0.9521 & 0.9756 \\
\hline 5. & 0.8231 & 0.9712 & 0.8346 & 0.9533 & 0.9624 \\
\hline 6. & 0.8221 & 0.9626 & 0.8239 & 0.9512 & 0.9742 \\
\hline 7. & 0.8229 & 0.9764 & 0.8317 & 0.9534 & 0.9681 \\
\hline 8. & 0.8125 & 0.9711 & 0.8366 & 0.9561 & 0.9756 \\
\hline 9. & 0.8321 & 0.9736 & 0.8278 & 0.9624 & 0.9612 \\
\hline 10. & 0.8149 & 0.9624 & 0.8289 & 0.9600 & 0.9782 \\
\hline
\end{tabular}

In this paper, our algorithm was evaluated in terms of sensitivity (Se), specificity (Sp), positive predictive value (Ppv), negative predictive value (Npv), and accuracy (Acc). Taking Table III into account, these metrics are defined as

$$
\begin{gathered}
S_{e}=\frac{T P}{T P+F N} \\
S_{P}=\frac{T N}{T N+F P} \\
P_{P V}=\frac{T P}{T P+F P} \\
N_{P V}=\frac{T N}{T N+F N} \\
A c c=\frac{T P+T N}{T P+F P+T N+F N}
\end{gathered}
$$

\section{B. Classification Accuracy}

The accuracy is measured by generating classifications for cases with known outcomes. In all cases, proposed ELM will produce the most accurate classifier than a model built with ANN and SVM technique. The proposed ELM technique perform well in huge datasets but the existing ANN and SVM technique does not perform well in huge datasets. From Table 3 , it is revealed that the proposed ELM has higher classification accuracy than the existing ANN and SVM techniques. For regression, it is found that ELM provides the highest accuracy.

Table 3: Classification Accuracy Comparison

\begin{tabular}{|c|c|c|c|}
\hline Images & ANN & SVM & Proposed ELM \\
\hline 1 & 89 & 91 & 96 \\
\hline 2 & 90 & 92 & 97 \\
\hline 3 & 86 & 88 & 98 \\
\hline 4 & 88 & 90 & 98.5 \\
\hline
\end{tabular}

\section{CONCLUSION}

Previous methods for blood vessel detection in retinal images can be classified into rule-based and supervised methods. This study proposes a method within the latter category. This method presents a new supervised technique for blood vessel detection in digital retinal images. This novel approach uses an Extreme Learning Machine (ELM) approach for pixel classification and calculates a 7-D vector comprises of gray-level and moment invariants-based features for pixel representation. The performance of the proposed approach is evaluated on the DRIVE and STARE databases. It is observed that the proposed approach provides significant output in terms of accuracy.

\section{REFERENCE}

[1] Diego Marín, Arturo Aquino, Manuel Emilio Gegúndez-Arias, and José Manuel Bravo, "A New Supervised Method for Blood Vessel 
Segmentation in Retinal Images by Using Gray-Level and Moment Invariants-Based Features", IEEE Transa. Med. imaging, Jan 2011

[2] R. Klein, S. M. Meuer, S. E. Moss, and B. E. Klein, "Retinal microaneurysm counts and 10-year progression of diabetic retinopathy," Arch.Ophthalmol., vol. 113, pp. 1386-1391, 1995.

[3] P. Massin, A. Erginay, and A. Gaudric, Rétinopathie Diabétique. New York: Elsevier, 2000.

[4] E. Ricci and R. Perfetti, "Retinal blood vessel segmentation using line operators and support vector classification," IEEE Trans. Med. Imag., vol. 26, no. 10, pp. 1357-1365, Oct. 2007.

[5] A. M. Mendonça and A. Campilho, "Segmentation of retinal blood vessels by combining the detection of centerlines and morphological reconstruction," IEEE Trans. Med. Imag., vol. 25, no. 9, pp. 12001213,Sep. 2006.

[6] J. Staal, M. D. Abràmoff, M. Niemeijer, M. A. Viergever, and B. v. Ginneken, "Ridge based vessel segmentation in color images of the retina," IEEE Trans. Med. Imag., vol. 23, no. 4, pp. 501-509, Apr. 2004.

[7] A. Hoover, V. Kouznetsova, and M. Goldbaum, "Locating blood vessels in retinal images by piecewise threshold probing of a matched filter response," IEEE Trans. Med. Imag., vol. 19, no. 3, pp. 203-210, Mar. 2000.

[8] Research Section, Digital Retinal Image for Vessel Extraction (DRIVE) Database. Utrecht, The Netherlands, Univ. Med. Center Utrecht, Image Sci. Inst. [Online]. Available: http://www.isi.uu.nl/Research/Databases/DRIVE

[9] STARE Project Website. Clemson, SC, Clemson Univ. [Online]. Available: http://www.ces.clemson.edu/

[10] T. Walter, P. Massin, A. Erginay, R. Ordonez, C. Jeulin, and J. C. Klein, "Automatic detection of microaneurysms in color fundus images," Med. Image Anal., vol. 11, pp. 555-566, 2007.

[11] C. Sinthanayothin, J. F. Boyce, H. L. Cook, and T. H. Williamson, "Automated localisation of the optic disc, fovea and retinal blood vessels from digital colour fundus images," Br. J. Ophtalmol., vol. 83, pp. $902-$ 910, 1999.

[12] M. Niemeijer, J. Staal, B. v. Ginneken, M. Loog, and M. D. Abramoff, J. Fitzpatrick and M. Sonka, Eds., "Comparative study of retinal vessel segmentation methods on a new publicly available database," in SPIE Med. Imag., 2004, vol. 5370, pp. 648-656.

[13] X. Jiang and D. Mojon, "Adaptive local thresholding by verification based multithreshold probing with application to vessel detection in retinal images," IEEE Trans. Pattern Anal. Mach. Intell., vol. 25, no. 1, pp. 131-137, Jan. 2003.

[14] G. G. Gardner, D. Keating, T. H.Williamson, and A. T. Elliott, "Automatic detection of diabetic retinopathy using an artificial neural network: A screening tool," Br. J. Ophthalmol., vol. 80, pp. 940-944, 1996.

[15] Guang -bin Huang,"Introduction to Extreme Learning Machine",workshop on Machine learning for Biomedical Informatics,Nov 2006.

[16] Qin-Yu Zhu,Chee-Kheong Siew,” Extreme Learning Machine: A New Learning Scheme of Feedforward Neural Networks", Neurocomputing, vol. 70, pp. 489-501, 2006.

[17] J. V. B. Soares, J. J. G. Leandro, R. M. Cesar, Jr., H. F. Jelinek, and M. J. Cree, "Retinal vessel segmentation using the $2 \mathrm{D}$ Gabor wavelet and supervised classification," IEEE Trans. Med. Imag., vol. 25, no. 9, pp. 1214-1222, Sep. 2006. 